\begin{tabular}{|c|c|}
\hline Citation & $\begin{array}{l}\text { D. Van Hertem, W. Leterme, G. Chaffey, M. Abedrabbo, M. Wang, F. Zerihun, M. } \\
\text { Barnes, "Substations for Future HVdc Grids: Equipment and Configurations for } \\
\text { Connection of HVdc Network Elements," in IEEE Power and Energy Magazine, vol. } \\
\text { 17, no. 4, pp. 56-66, July-Aug. 2019. }\end{array}$ \\
\hline Archived version & @2019 IEEE \\
\hline \multirow[t]{2}{*}{ Published version } & DOI: 10.1109/MPE.2019.2909006 \\
\hline & $\begin{array}{l}\text { URL (IEEE Digital Library): } \\
\text { https://ieeexplore.ieee.org/document/8739080 } \\
\end{array}$ \\
\hline Author contact & $\begin{array}{l}\text { Dirk Van Hertem } \\
\text { dirk.vanhertem@esat.kuleuven.be } \\
\text { tel: }+3216321020\end{array}$ \\
\hline
\end{tabular}




\title{
Substations for Future HVdc Grids: Equipment and Configurations for Connection of HVdc Network Elements
}

\author{
Dirk Van Hertem, Willem Leterme, Geraint Chaffey, Mudar Abedrabbo, Mian Wang, Firew \\ Zerihun, Mike Barnes
}

With increased integration of renewable energy generation, high-voltage direct current (HVdc) will become more prevalent in the power system. Anticipated annual growth rates are in the range of $7-10 \%$. While most systems in operation and under construction are point-to-point connections, the first multiterminal HVdc systems have already been commissioned. As a next step, HVdc grids are being considered as a cost-effective solution to transmit power. These $\mathrm{HVdc}$ grids are expected to gradually develop from existing point-to-point links, mirroring the development of ac grids throughout the $20^{\text {th }}$ century. Such HVdc grids will be an integral part of the power system, operating as a separate transmission layer of the future hybrid ac/dc network.

DC substations will constitute an integral part of HVdc grids. These substations will differ from the typical dc converter station of existing point-to-point connections and from ac substations. In this article, we discuss the similarities and differences between dc substations and their ac counterparts in terms of layout, technologies, and requirements.

\section{HVdc Technology}

$\mathrm{HVdc}$ is a well-established technology for grid reinforcements that avoids some of the disadvantages associated with ac transmission upgrades (increases ac system fault level, requirements for reactive power, and synchronized ac networks) by transmitting electric energy over dc circuits. It has been used commercially for more than 60 years, mainly to transmit bulk power over large distances and connect asynchronous ac systems, and it is a favorable choice when long (undersea) cable connections are needed.

$\mathrm{HVdc}$ transmission utilizes power-electronic converters to transform ac to dc and dc to ac. The converters utilize either thyristor or insulated-gate bipolar transistor (IGBT) power electronic switches. Thyristors are used in line-commutated converters (LCCs), the traditional HVdc technology, while IGBTs are used in voltage-source converters (VSCs). While both technologies can provide fast control of active power and power transmission using overhead line or cable systems, there are differences between the two. On the one hand, LCCs are a more mature, cost-effective, and efficient technology that is available for very large power ratings (on the order of $10 \mathrm{GW}$ ). Alternatively, VSC HVdc applications offer several advantages, including a smaller overall footprint, fast active power reversal capability, flexible and independent control of reactive power, easier integration into weak power systems, and use of extruded cables, for instance, based on cross-linked polyethylene (XLPE).

Since the turn of the century, HVdc has seen a revival. In countries such as China, India, and Brazil, LCC HVdc installations transport bulk power over long distances, fulfilling a need caused by fast economic growth. At the same time, the development of VSC technology led to new applications that include using submarine cables to interconnect oil platforms and offshore wind farms with onshore facilities and underground cable and overhead line applications to provide land-based links.

Recent advancements in VSC technology have enabled the development of an HVdc grid with three or more converters connected in parallel. Several such systems have been developed or are under construction. Whereas point-to-point systems are typically developed as projects 
using an engineering-procurement construction procedure, future HVdc grids will, in many cases, be developed gradually, requiring interfaces among projects from different vendors.

Although LCC HVdc grids or hybrid VSC/LCC HVdc grids are possible, this article focuses on VSC HVdc grids, their substation layout, and how they differ from their ac counterparts. HVdc substations consist of a dc switchyard connecting multiple lines or cables and, possibly, one or more converter stations. As with ac systems, devices can be categorized as primary equipment, which carries the power, and secondary equipment, which provides interfacing and control.

\section{Primary Equipment}

In a future dc substation, the primary equipment could consist of various components for power transfer, protection, and switching capabilities for system reconfiguration. Prospective components include ac/dc converters, circuit breakers and other switchgear, cables, lines and their terminations, means of discharging cables, and equipment to control power flow, as shown in Figure 1.

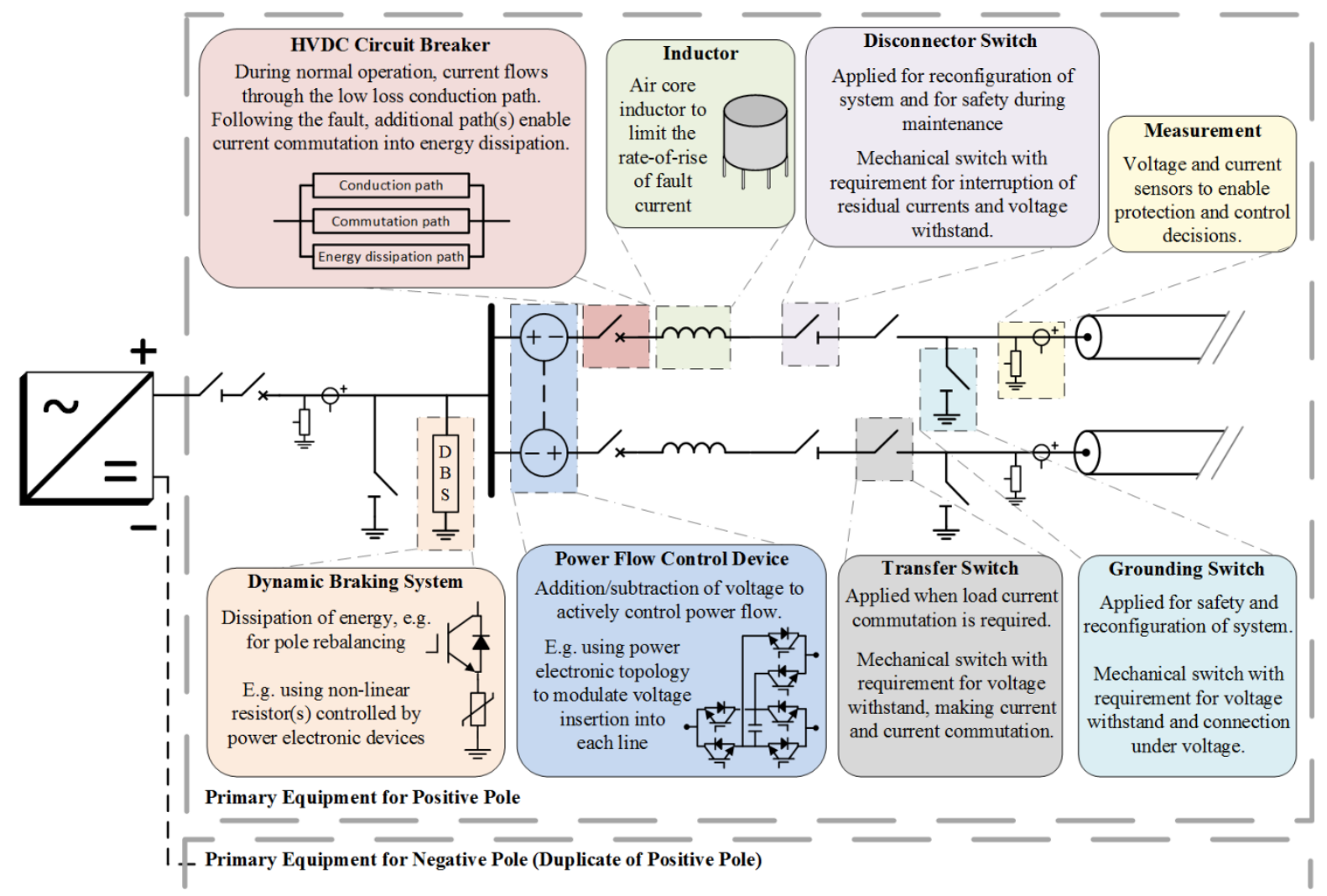

figure 1. A prospective primary-equipment layout per pole for a symmetric monopolar system. Note that not all equipment is required in all substations and that the configuration is given as an example and is not indicative of an optimal system layout.

\section{AC/DC Converters}

Converting ac to dc is an essential requirement for the power system of the future, enabling the connection of HVdc systems to existing ac transmission networks for bulk energy transfer. A VSC station consists of the VSC valve hall, in which the electronic equipment is placed, as well as phase inductors and measurement equipment. The ac transformers and switchyard are typically placed outside the VSC area of the substation.

Various VSC technologies can be used for ac/dc conversion in multiterminal systems, which can be roughly classified into two main types: dc-fault feeding (for example, a half-bridge 
modular multilevel converter) and dc-fault blocking (for example, a full-bridge modular multilevel converter). Both types can control the dc-side current during normal operation; however, they can be differentiated by their dc-fault response.

The dc-fault response by the converter can have a significant influence on the design of other primary equipment within the HVdc substation. Following a dc fault, dc voltage collapse rapidly propagates across the network; the current rises quickly as the rate of rise is limited by the otherwise relatively low inductance, as opposed to the relatively high impedance in an ac grid. Consequently, the power-electronic devices in affected fault-feeding converters are switched off (blocked), protecting them from damage without stopping fault-current infeed from the ac grid. These topologies, thus, require other equipment to interrupt the fault current. By contrast, fault-blocking converters do not need to rely on other equipment to interrupt fault currents, as they have the capability to either block or control the current contribution through the converter.

\section{Circuit Breakers and Switchgear}

Within a dc substation, there are several requirements for switching, which can be grouped according to functionality: grounding, disconnecting, current commutation, and current interruption. Fault-current interruption devices at the dc side (HVdc circuit breakers) are essential to achieve selective protection of multiterminal HVdc systems by isolating only the faulty element.

The requirements for interrupting a dc current lead to challenges with HVdc circuit breakers not observed in ac systems. The absence of naturally recurring current-zero crossings requires the HVdc circuit breaker to drive the dc fault current toward zero and absorb the energy that remains in the system at the moment the circuit breaker opens. To perform these functions, an HVdc circuit breaker typically makes use of parallel paths with auxiliary circuits to enable current commutation and energy absorption. In addition, high switching speeds require fast mechanical operation or power-electronic switches in the circuit. Recently proposed HVdc circuit breakers can operate within $2-10 \mathrm{~ms}$, an order of magnitude faster than an equivalent ac device.

The dc-fault current can reach destructive values within milliseconds and exceed the HVdc circuit breaker's interruption capability. Therefore, series fault-current limiters (for instance, a line inductor) may be required to limit the rate of rise of fault current. Such inductors, for conducting a dc current, are air cored, physically large, and require additional space to avoid interference with neighboring equipment.

Apart from dc fault-current-interruption devices, several other important HVdc switching applications operate 10-100 times slower than a typical dc fault-current interruption. Applications include grounding a cable, disconnecting a converter, reconfiguring a dc substation, and isolating a faulty element (for example, following fault-current interruption using fault-blocking converters). For these operations, disconnector, grounding, and transfer switches can be used.

\section{Energy Dissipation}

There is often a requirement for discharging cables and overhead lines, either for safety when taking a line out of service for maintenance, for dissipating excess energy during a momentary fault (for example, from a wind farm, for which power cannot be instantaneously decreased), or for balancing unequal pole voltages in a symmetric monopolar network.

For planned maintenance, a grounding switch can be utilized, which could take seconds to discharge the line and reopen. This is acceptable given that this operation has no stringent time 
constraints. In the case of pole balancing, however, there is a requirement to resume power flow as quickly as possible (in the post dc-fault case) or continue normal operation (for balancing in nonfault conditions). Different solutions based on power electronics or nonlinear resistors have been suggested to meet these demands.

\section{Power-Flow Control Devices}

Controlling the power flow enables the use of any power system at its full capacity. The topology and configuration of the passive network and the voltage at each node determines power flow unless active devices are applied. While flexible ac transmission systems already dynamically control power flow in ac grids, there are currently no commercially available products for $\mathrm{HVdc}$ grids.

In an HVdc grid, the voltage difference and resistance between two nodes determine the power flow through a transmission line. Therefore, the power flow can be controlled by adding resistance (although incurring additional losses would be unattractive) or modifying the node voltages at each end of a line. On a meshed system, for which each node voltage affects the power flow on multiple lines, it is not possible to maximize the power flow on all lines, resulting in some lines inevitably being underutilized. Some proposed solutions insert a controllable voltage in series with a line, with the absorbed energy transferred to an adjacent line using an equivalent but opposing voltage. The voltage requirement would be of similar magnitude to the voltage drop across the line, small compared with the pole-to-ground voltage.

DC power-flow control devices are still in a conceptual phase as they will influence losses and system reliability and could be expensive. Within the substation, these devices might consume considerable space and add to the complexity of system design and operation.

\section{DC/DC Converters}

Transferring energy between different dc voltages requires a different solution compared to the ac transformer, which provides effective passive-voltage transformation. While there are no $\mathrm{HVdc} \mathrm{dc} / \mathrm{dc}$ converters in operation today, there could be benefits in connecting HVdc systems of different voltages. An example would be increasing operational flexibility by interconnecting networks that were built at different times. DC/DC converters could either be directly connected or provide galvanic isolation when using an intermediary ac stage. Furthermore, they would also have the ability to control power flow. A dc/dc converter might also be able to prevent a fault from propagating across the network, although this may come at additional costs.

\section{Bushings}

Bushings, which are insulation devices, allow the HV conductor to pass safely through the grounded tank and isolate energized primary components and substation buswork from the ground. A bushing consists of a conductor surrounded by insulation material, which is typically made from porcelain.

Design procedures from the insulation system used in HVac bushings cannot be directly applied to HVdc bushings due to differences in electric-field distributions between ac and dc systems. Additionally, various connected components in dc substations employ different types of bushings. For example, the design of wall bushings, which connect the valve hall with other primary components, is different from that of the bushings used in HVdc circuit breakers.

\section{Secondary Equipment}


Secondary equipment in dc substations can be expected to require more computational power and communications than in ac substations, given the greater complexity of the many control and protection functions needed to operate an HVdc grid.

\section{Measurement Devices}

Conventional instrument transformers based on electromagnetic-induction technology are the most widely used primary sensors in ac grids. The bandwidth of such transformers is usually limited to a few kilohertz, as, beyond these frequencies, the conversion ratio of these devices is nonlinear due to resonances and capacitive couplings. For HVdc applications, nonconventional instrument transformers (NCITs) must be used.

Zero-flux current sensors, combined shunt and Rogowski coils, and fiber-optical current sensors can be used to measure currents in HVdc grids. Resistive-capacitive (RC) voltagedivider technology is most commonly adopted for $\mathrm{HVdc}$ voltage measurement. The future trends of NCITs for HVdc grids include compact and possibly integrated options through modern technologies, such as RC dividers for gas-insulated switchgear (GIS) and integrated optic sensors. Compared to ac substations, a larger number of current and voltage instrument transformers are likely to be used in dc substations. This is because these substations contain more components that must be controlled or protected, such as dc/dc converters, dc lines, inductors, and HVdc circuit breakers. In a digital substation, instrument transformers are interfaced with merging units, which digitize the measured quantities and send the sampled values to control and protection devices via peer-to-peer communication or multicasting to multiple subscribers.

\section{Control}

HVdc grid control ensures stable operation by controlling the dc voltage, which presents itself as a global parameter within the grid. Many dc-voltage control approaches are expected to be used for HVdc grids, which can be divided into centralized and distributed approaches. In the centralized approach, one converter responsible for the control of direct voltage acts as a dc slack bus, while the other converters regulate active power. This approach is not suitable for larger HVdc grids because dependence on a single slack converter could lead to reliability issues. In the distributed approach, all the converters in the HVdc grid control share the active power balance by employing a voltage-droop scheme. However, this requires coordination among substation controllers and HVdc grid controllers. The distributed approach is more appropriate for larger HVdc grids but can also be used for smaller HVdc grids if needed.

$\mathrm{HVdc}$ substation controllers are relatively fast regulators that include primary voltage-power control in each converter station in the HVdc grid. This control may take the form of voltagedroop characteristics and, thereby, enable automatic power sharing among several converters without depending on external communication. This approach is similar to power-frequencydroop control in ac grids. The substation controllers have time constants on the order of a few to tens of milliseconds and, in turn, require high-bandwidth communication channels. The substation controllers might consist of pole- or station-level controllers responsible for setting the active power, reactive power, and ac or dc voltage orders to the outer controllers of each converter.

The HVdc grid controllers are higher-level equipment; this equipment includes the secondary and tertiary control. The secondary control is responsible for correcting the active-power set points after a contingency, with a response time of a few seconds. The HVdc grid tertiary control is responsible for optimal power flow that considers the state and requirements of the combined ac and dc grids. These controls might be implemented as a master at a single physical location at any one time or as a distributed spread across different physical locations. 
The HVdc grid controller provides set points to the local substation controllers. These controllers generally have slower response times, tens to hundreds of milliseconds or longer, and require relatively low communication channel bandwidth. The control signals and set points are communicated to the converters via dedicated communication channels, which might allow operator interactions from one central location or from multiple locations within the system during steady-state operations, or from automatic set point or control-parameter modifications during transients. The functionality of the $\mathrm{HVdc}$ grid controller depends on the design philosophy of the particular grid, the structure of the ac grid, the regulatory framework in place, and network codes.

$\mathrm{HVdc}$ converters that are part of $\mathrm{HVdc}$ grids should have plug-and-play characteristics to guarantee effective operation in a multivendor environment. This requires the converters to handle a loss of communication by relying on local measurements via autonomous converter control. These control perspectives have a direct consequence for the control hierarchy in the system and the communication system requirements.

\section{Intelligent Electronic Devices for Protection}

Relay technologies have evolved from early electromechanical to static, digital, and numerical relays. Modern numerical relays, or so-called intelligent electronic devices (IEDs), use one or more digital signal processors optimized for real-time signal processing and capable of running a wide range of protection functions. Numerical IED performance greatly surpasses that of other technologies in terms of accuracy, range of parameter settings, built-in communications, and multiple functionalities that include monitoring and self-diagnostics.

The main differences between ac and HVdc IEDs encompass the protection algorithm, operational speed, and the input/output requirements. AC IEDs are typically interfaced only with their associated ac circuit breakers and coordinated among local and remote terminals. However, HVdc IEDs might be required to carry out more complex functions to coordinate protection and restoration, such as sending fault detection and fault-clearing signals to the converter or pole rebalancing equipment. In addition, $\mathrm{HVdc}$ circuit breakers are likely to have more intelligent functions, such as self-diagnostics, leading to potential coordinated protection through the communication of breaker failures to nearby IEDs.

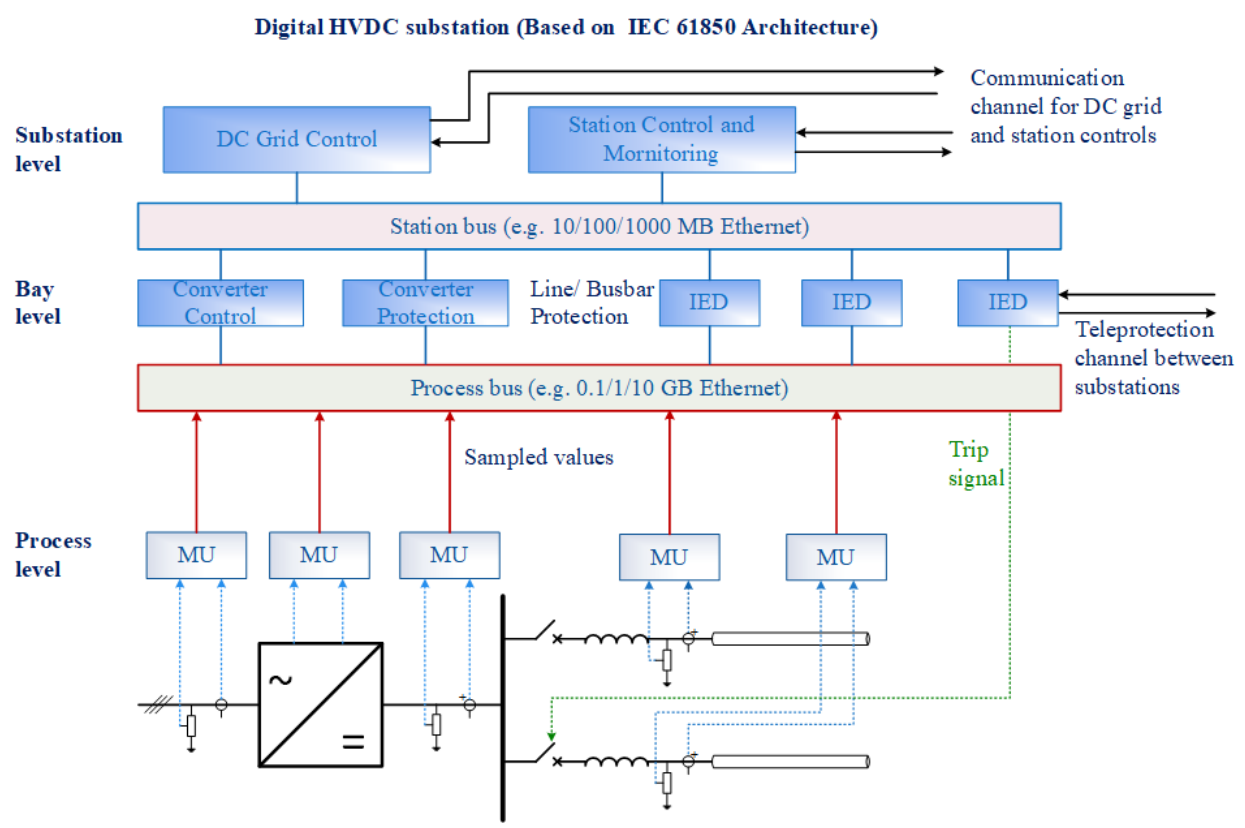


In Figure 2, a decentralized protection configuration is given in which each IED is responsible for protecting one component. A centralized protection configuration is also an option, where all protection functions are implemented physically on one computer. The benefit of the former is higher reliability because the failure of one IED most likely leads to a backup IED operation. The latter likely provides simplified design and protection coordination; however, failure of the central protection might lead to unacceptable consequences.

\section{Communication}

Since existing HVdc links were built as turnkey projects by a single company, communication protocols for control and protection are, to a large extent, vendor specific. For the development of large meshed HVdc grids, standardization is essential to ensure interoperability between vendor equipment. Similar standards, such as IEC 61850 for digital substations, IEC 61869 for instrument transformers, and IEC 60834 for teleprotection, could also be adapted for HVdc applications. However, the required communication speed and channel bandwidth are likely to be higher for HVdc grids compared to ac grids.

The protection and control architecture of a future dc substation can, from a communication perspective, adopt a similar structure as defined by IEC 61850 (Figure 2). Communication can be optimally designed for the whole dc substation using technologies such as fast Ethernet. The required performance of the communication for ac grids is 80 samples/cycle and $3 \mathrm{~ms}$ for sampled values and generic object-oriented substation event message, respectively. This requirement for $\mathrm{HVdc}$ grids should be on the order of $100 \mathrm{kSamples} / \mathrm{s}$ and hundreds of microseconds to fulfil the requirement of fast control and protection.

Communication between remote dc substations includes dispatching, control signals, and teleprotection signals. Digital communication can be accomplished using fiber-optic channels. Control set points and parameters at the dispatching level require the least bandwidth and communication speed. Communication for teleprotection, and, in particular, line-differential algorithms, requires the highest bandwidth and communication speed.

\section{Digital DC Substation}

Existing ac substations are undergoing a transformation to digital substations employing IEC 61850 standards in their protection and control architecture. Future dc substations can also employ a similar design principle, digitizing the primary signals (such as current and voltage measurements), switchgear-position status and control, and sending digital signals to the protection and control devices using fast communication channels. An example is shown in Figure 2.

\section{Substation Design Considerations}

Although there are more than $100 \mathrm{HVdc}$ systems in operation and multiterminal HVdc systems have been built, the concept of dc substations is not well established. A substation in a VSC HVdc grid is different from an ac substation or VSC HVdc point-to-point connection. A dc substation contains the bays of incoming cables, transmission lines, and converter stations. The bays include line and cable terminations, switchgear, measurement equipment, and other secondary equipment for protection and control. Within the substation, the bays are connected to busbars in a particular arrangement.

DC substation design may differ considerably from ac substation design, given the increased dimensions and cost of dc-side fault-clearing equipment and different options for protection and configuration and grounding of the HVdc grid. These aspects influence dc substation design because they affect the balance between cost and expected availability as well as considerations for the future growth of the HVdc grid. We now briefly discuss factors affecting 
substation design choices for HVdc grids, including configuration, grounding, protection philosophies, and technology.

\section{HVdc Configuration and Grounding}

The configuration of an HVdc grid influences the substation layout because it establishes the number of bays per connected circuit. DC substations must accommodate a single pole or two poles for each circuit, with the addition of a possible ground connection and metallic return, as illustrated in Figure 3. The case of one connected bay per circuit results in the asymmetric monopolar configuration [Figure 3(a)]. In this case, the current flows through one pole and returns via the ground. This option is often not permitted for environmental reasons. Two bays per connected circuit causes either an asymmetric monopolar configuration with metallic return [Figure 3(a)], which is low-impedance grounded, or a symmetric monopolar configuration [Figure 3(b)], where the dc side is ungrounded or high-impedance grounded. Three bays per connected circuit brings about a bipolar configuration where one of the conductors is lowimpedance grounded and acts as a metallic return conductor [Figure 3(c)].

The grounding of the HVdc grid influences dc substation design by determining the expected fault current and overvoltage levels and, hence, the required substation equipment ratings. When an asymmetric monopolar or bipolar configuration is used, the voltages during the fault remain limited, yet the prospective fault current is high. In systems with symmetric monopolar configurations, the overvoltage on the healthy pole could reach up to two times the nominal voltage during faults in the absence of overvoltage protection, whereas the steady-state fault current would be zero. The peak fault current and peak voltage that can occur during dc short circuit faults depend on the speed of fault clearing and fault-current-limiting equipment used within the HVdc grid. These aspects enter the cost equation of the substation as a whole. A tradeoff must be made between dc-fault current or overvoltage- level reduction and the cost of fault-clearing equipment.

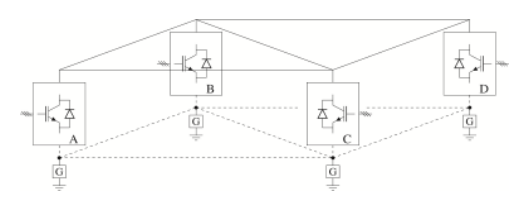

(a)

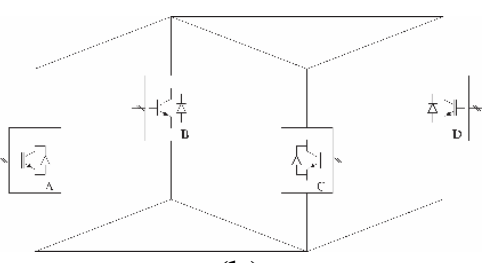

(b)

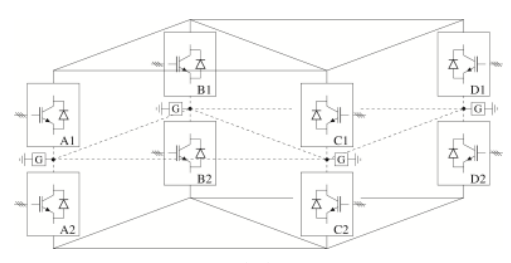

(c)

figure 3. Configurations and grounding for HVdc grids: (a) asymmetric monopolar, (b) symmetric monopolar, and (c) bipolar.

\section{HVdc-Protection Philosophies}

The amount of protection equipment required in a dc substation depends on the protection philosophy of the HVdc grid. To avoid a large number of potentially expensive HVdc circuit breakers, protection philosophies for HVdc grids may differ from the traditional ac systemprotection approach. In ac systems, protection zones are set for each component (line, transformer, bus, and so forth) to protect the system selectively. The consequence of this approach is that, in ac systems, circuit breakers are typically placed such that they can independently interrupt currents in each line terminal. This may not be the best approach for HVdc grids. An HVdc grid can adopt a nonselective, partially selective, or fully selective protection philosophy, which, upon the occurrence of a fault, clears the entire grid, a subpart, or only the faulted component, respectively.

In the nonselective case, equipment capable of interrupting fault currents is only placed at the boundaries of the HVdc grid (for instance, the ac/dc converters), making it a single protection zone for fault clearing. This results in the entire HVdc grid being de-energized before the 
faulted component is isolated under near-zero voltage and current conditions. After isolating the component, the rest of the HVdc grid is energized again. The equipment needed in the transmission line bays to isolate the faulted component may be mechanical switches, such as disconnector and fast disconnector switches.

In the fully selective case, which is similar to ac-system protection, all substation bays require equipment capable of interrupting fault currents to be able to individually isolate faults on every component of the HVdc grid. Because HVdc circuit breakers are generally larger than their ac counterparts and require the use of current-limiting equipment, the dc substation layout is expected to differ considerably from ac substation layout.

In the partially selective case, the protection zones divide the HVdc grid into subgrids, encompassing multiple lines and converters. The zones are within the HVdc gr id bounded by fault clearing equipment (for example, HVdc circuit breakers and dc/dc converters), which quickly isolate the faulted zone from the healthy ones. Similar to a nonselectively protected $\mathrm{HVdc}$ grid, the faulted zone is de-energized and the faulted component isolated using mechanical switches. This protection philosophy relies on two types of equipment: 1) mechanical switches without fault-current interruption capability in transmission line bays that do not connect protection zones and 2) equipment with fault-current interruption capability in bays that provide the interconnection between two protection zones.

\section{Busbar Arrangements}

The protection choice determines the required equipment within the substation and affects the busbar arrangement. In selectively protected HVdc grids, the balance of availability versus cost will likely result in a different busbar arrangement compared with nonselectively protected grids. In the former case, HVdc circuit breakers in each substation bay cost more than the disconnectors or switches in the latter case. Similar to existing ac substation layouts, the dc substation busbar arrangement can adopt a single bus, a double bus-double breaker, a mainand-transfer arrangement, a double bus-single breaker, a ring bus or breaker, and a half scheme. The schemes providing a higher availability generally require a higher number of switches. The use of HVdc circuit breakers in the substation bays substantially increases the cost and the size of the substation. For selectively protected HVdc grids, the relative location of the circuit breakers and fault-current-limiting equipment may need careful determination to avoid creating damaging overcurrents in the circuit breakers, for example, because of the discharge of the connected cables. Figure 4 provides examples of dc busbar arrangements, where care has been taken to avoid a direct connection between the circuit breaker and the transmission line.

While it is clear that the design of a dc substation will depend on the adopted protection philosophy, it will also hinge on the anticipated growth of an HVdc grid and a potential associated change of philosophy. Small-scale HVdc grids will be protected in a nonselective way, as is done for the existing multiterminal systems, whereas larger-scale HVdc grids will show an increasing need for dc-side selectivity. DC substation layout should provide sufficient space for fault-clearing equipment, such as HVdc circuit breakers, in case the HVdc grid is expected to grow.

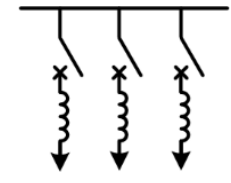

(a)

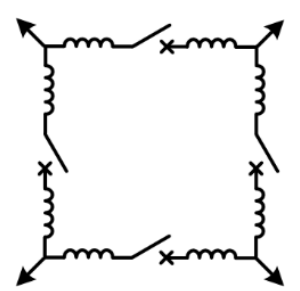

(b)

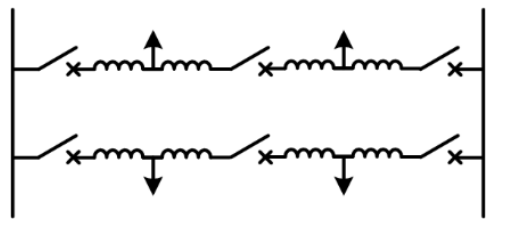

(c)

figure 4. An example of busbar topologies and arrangements for breakers and current-limiting inductors: (a) a single busbar/single breaker, (b) a ring bus, and (c) a breaker-and-a-half scheme. Measurements and disconnectors are not shown. 


\section{Substation Technology}

The choice of substation technology will influence its layout because GIS technologies allow for reduced dimensions compared with air-insulated switchgear technologies. Although GIS is a mature technology in ac grids, it is not widely used for HVdc. The ac GIS designs cannot be transferred straight to dc due to the differences in electric-field distributions. Currently, GIS technology for dc applications is under development for voltage levels appropriate for future HVdc grids. The first offshore application of dc GIS will be for the DolWin6 project in Germany; at present, measurement technology, such as RC dividers for GIS, are also being developed and tested.

\section{Offshore Substations}

HVdc grids are expected to play a key role in future offshore grids requiring specific installations on platforms where space and volume are precious. Since these substations are located far from land, platform size and system maintenance dominate design considerations and cost. DC substations would typically be a long distance from shore because ac technology would be used for shorter distances. Although the break-even distance varies from site to site, it is typically quoted as being around $100 \mathrm{~km}$. A dc substation far offshore would need to be housed in a large protective structure with a controlled internal environment that shields the equipment against temperature variation, weather, wind, and waves.

Figure 5 shows a typical offshore HVdc housing on a raised platform, which is many stories high and the size of a sports field. Substations have been constructed in the relatively shallow waters off the northwestern European coast where engineers use fixed foundation legs on the seabed. The civil engineering and installation expenses dominate the overall costs of such platforms and lead to great demand for compact designs that minimize the station size. As a result, all offshore dc substations have used VSCs and are likely to continue doing so because of their smaller footprint compared with LCCs. An alternative solution is the construction of artificial islands, but examples of this do not exist yet.

Considerable work is being undertaken to reduce the size of the housing (topside), which would further lower its cost. It would also decrease the size and cost of the foundation. The size of lifting vessels could be lessened which, given the limited supply of very large lifting vessels, could also potentially reduce construction lead time and costs.

The other major cost factor, maintenance, results from the challenges of transporting large equipment and personnel to a substation platform a long distance offshore. Several factors that must be considered include the following:

1) Weather: Transporting large pieces of equipment to a platform in poor weather conditions is troublesome. The delivery of replacement parts will be constrained by available weather windows.

2) Logistics: For the transportation of smaller plant pieces, parts, and technicians, a helicopter may be feasible. Suitable vessels and crew must be procured for large equipment. Large equipment is designed to be very reliable; if it fails at all, it may be just once during the 20-plus-year life of the HVdc system. Despite the equipment's high reliability, procuring and managing special vessels and crews would be required on the rare occasions of forced outages.

3) Spare parts: Spare equipment inventories for large plants may be prohibitively expensive. Spare parts may need to be manufactured (or existing plant equipment repaired on site).

4) Platform access: The part to be replaced may need to be extracted from the platform, and this may affect the layout of items in the substation, complicating electrical design. 
5) Revenue loss: While a dc substation is out of service, the power of the wind farm it connects to would be blocked or curtailed.

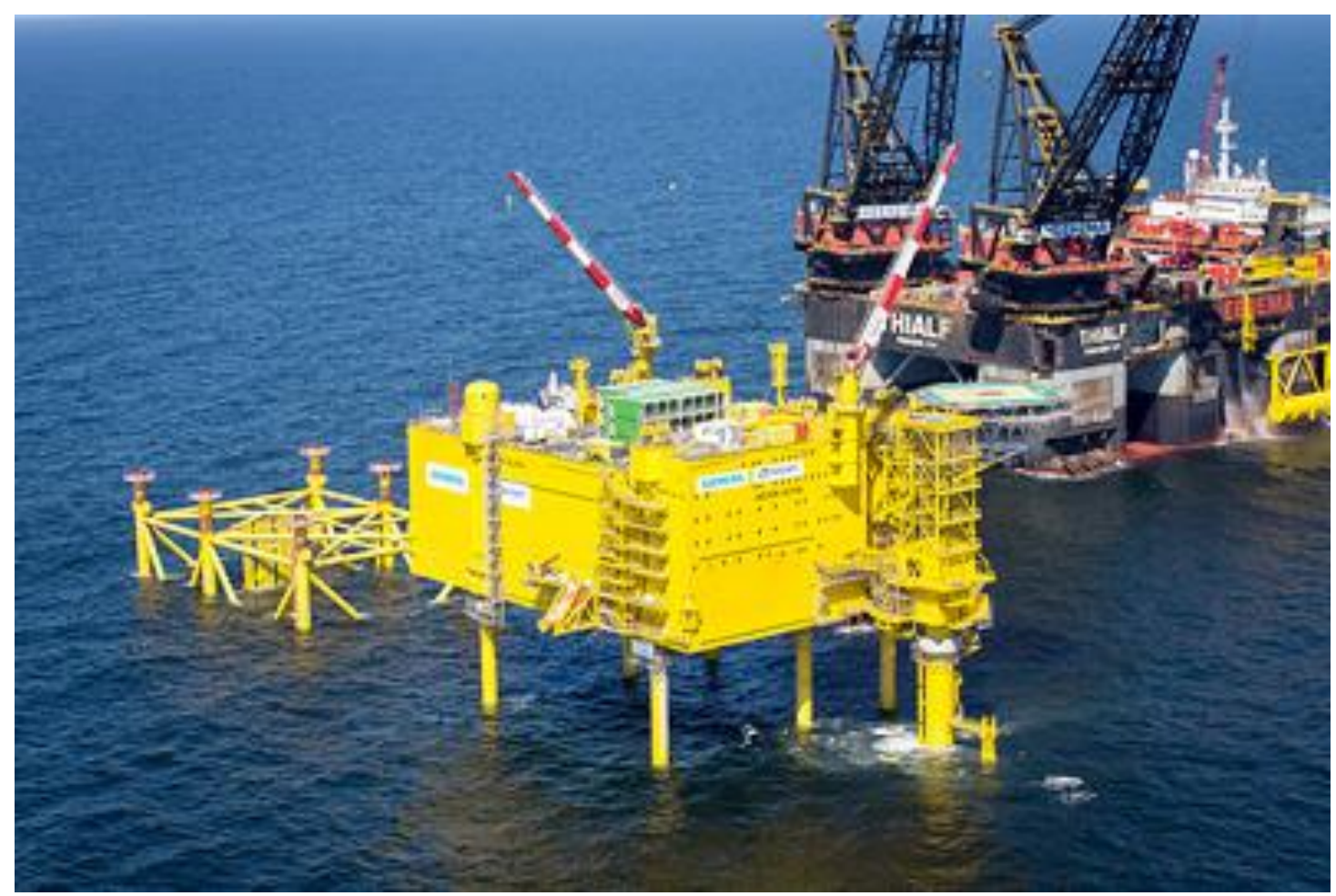

figure 5. The HelWin 2 foundation next to the HelWin 1 platform. (Used with permission of Siemens, www.siemens.com/press.)

Historically, operations and maintenance considerations have led to different onshore and offshore system designs. Figure 6 shows a simplified single-line diagram of a two-level, pointto-point link. The onshore substation design optimizes the system's electrical performance. The connection transformer has a tap changer to best match the network ac voltage to the converter. Since physically large transformers are essential components of a plant and including a tap changer decreases a plant's reliability, offshore connection transformers do not have a tap changer. A decrease in the offshore converter's efficiency is accepted to obtain lower costs in overall operations and maintenance.

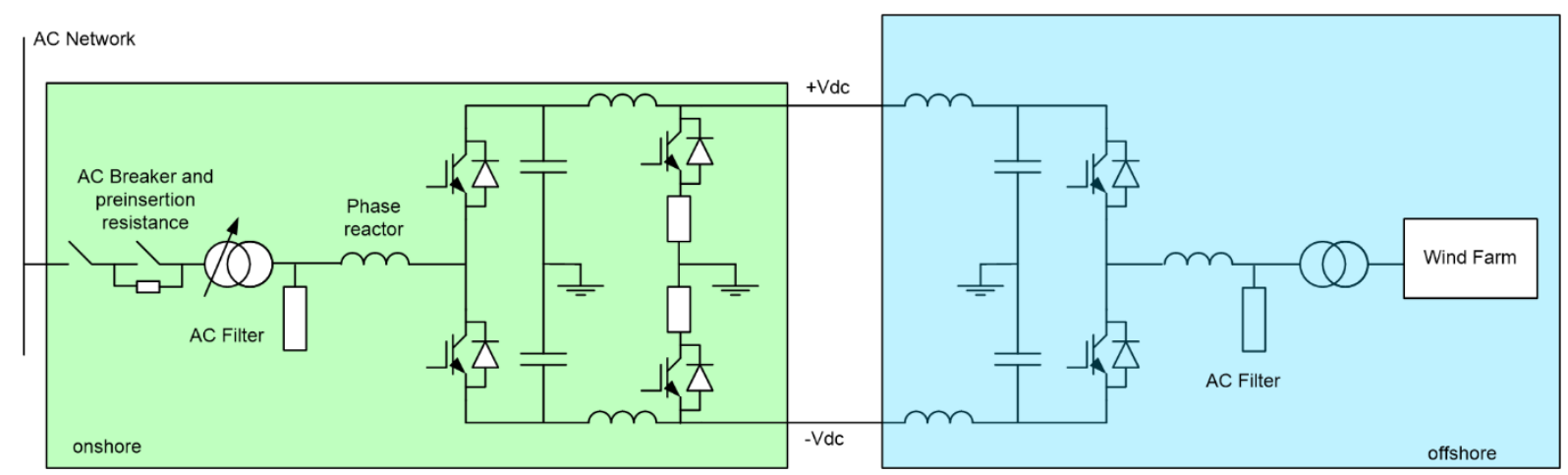

figure 6. A point-to-point, representative, single-line diagram of an HVdc two-level substation (based on published data from the BorWin 1 HVdc offshore platform).

Where possible, equipment is not located offshore. Figure 6 shows the onshore location of dc chopper resistors, which are used to ensure that the dc-connected cables do not experience overvoltages. The space to house them is not part of the offshore substation, again reducing costs. 
Since multiterminal substations would be more complex and larger, locating them offshore would increase the size and cost of offshore substations. The greater complexity would also increase the potential for component failure. The application of $\mathrm{HVdc}$ breakers, with their large and complex components, would further increase substation costs. These increased costs are particularly high when the multiterminal dc substations are located offshore on a platform, potentially limiting the development of such substations.

There have been substantial investments in VSC HVdc reliability. Converters now employ modular multilevel designs using several submodules. As shown in Figure 7, each submodule is about the size of a large suitcase and can be replaced individually. A converter can be designed with redundant submodules; should one fail, it can be switched out and replaced with another online.

The design of offshore converters requires special expertise. Even with $99 \%$ efficiency, a 500MW station still requires cooling systems to handle up to $5 \mathrm{MW}$ of heat. In addition to thermal design, electrical-insulation design is a key factor. Sufficient clearance must be left between components of the converter and the topside structure to ensure proper electrical isolation. This means that much of the structure inside the topside is clear air. The authors anticipate the first applications of DC GIS solutions to be deployed offshore. While some onshore VSC HVdc designs use voltages up to $500 \mathrm{kV}$, present offshore installations are limited to $\pm 320 \mathrm{kV}$. This is consistent with the voltage ratings of the XLPE subsea cables that were available at the time of this writing.

Major manufacturers have undertaken a large number of offshore substations, and these have been functioning successfully for a number of years. The authors anticipate further progress in reducing station size and cost. In maintenance, the use of robots, already seen in some onshore substations and wind turbines, will undoubtedly transfer to offshore environments.

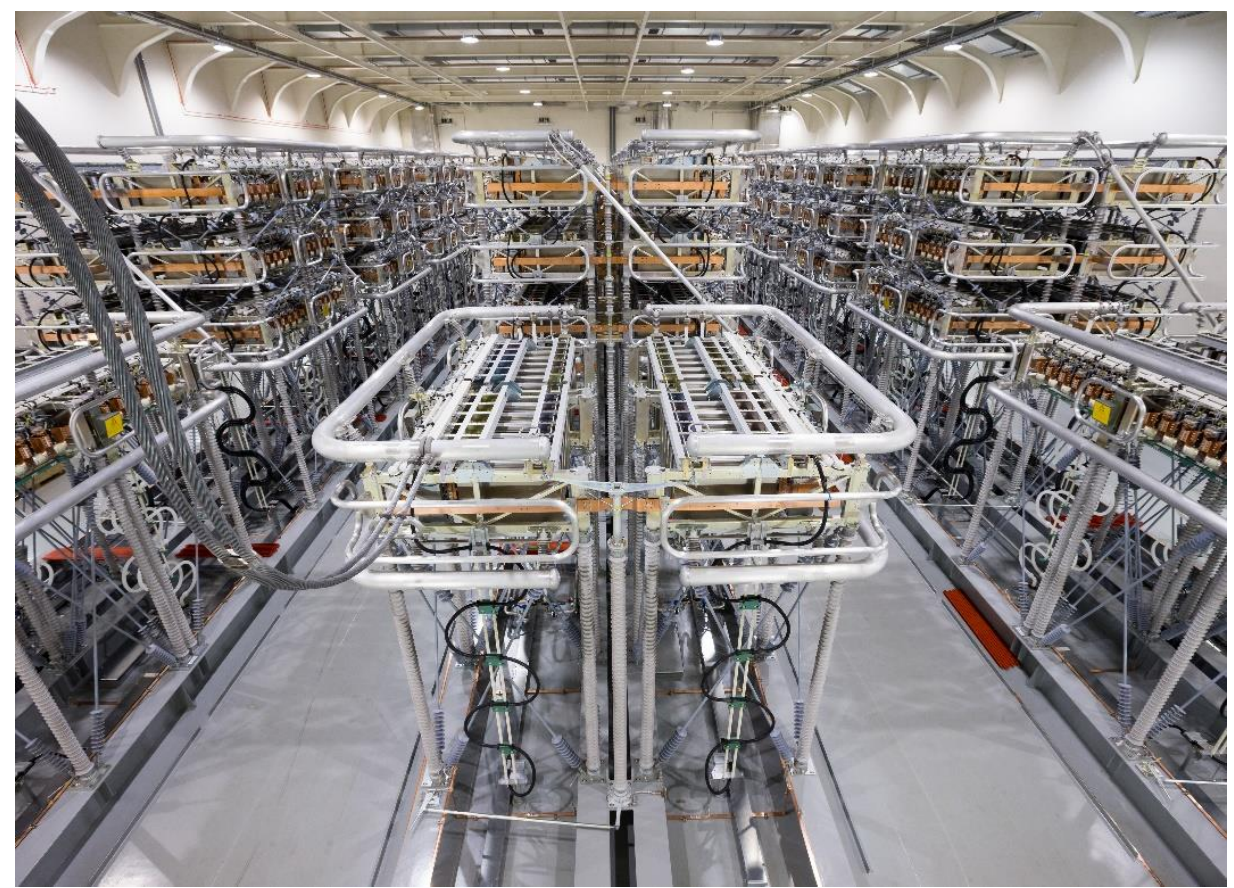

figure 7. The interior of the SylWin 1 offshore substation. (Used with permission of Siemens, www.siemens.com/press.)

\section{Summary}

Although the design principles for ac substations, such as substation layout, are, in theory, applicable to the design of dc substations, fundamental differences exist and depend on the choices made in system topology, control, and protection. First, the HVdc grid topology 
determines the number of transmission lines to route within a dc substation. Second, the primary and secondary equipment required within the dc substation, and its characteristics, depends on the adopted control and protection philosophy. In addition, the location of the substation (for example, on- or offshore) influences the choices made in construction and insulation technology (for instance, air or gas insulated). Recent technological developments indicate that most building blocks for future HVdc grids are in place or advancing toward practical realization. Nevertheless, the construction of a reliable and cost-effective HVdc grid will require engineers to consider the many options for system protection, operation, and control, and the implications that these have on the practical realization of the underlying $\mathrm{dc}$ substations.

\section{For Further Reading}

M. Barnes, D. Van Hertem, S. P. Teeuwsen, and M. Callavik, "HVDC systems in smart grids,” Proc. IEEE, vol. 105, no. 11, pp. 2082-2098, Nov. 2017.

CIGRÉ Joint Working Group B4/B5-59, "Local control and protection of HVDC grids," CIGRÉ, Paris, France, technical brochure, 2018.

CIGRÉ Joint Working Group A3/B4.34, "Technical requirements and specifications of state-of-the-art HVDC switching equipment," CIGRÉ, Paris, France, technical brochure, 2017.

D. Van Hertem, O. Gomis-Bellmunt, and J. Liang, HVDC Grids: For Offshore and Supergrid of the Future. Hoboken, NJ: Wiley-IEEE Press, 2016.

V. Akhmatov et al., "Technical guidelines and prestandardization work for first HVDC

grids," IEEE Trans. Power Del., vol. 29, no. 1, pp. 327-335, Feb. 2014. doi: 10.1109/ TPWRD.2013.2273978. 